\title{
Glomerulitis Inducida en Ratones Mediante Inmunocomplejos (IC). Efecto del Líquido Amniótico (LA) Sobre la Depuración de IC y la Histopatología Renal
}

\author{
Dr. Jorge Cerda, M.D. ${ }^{1}$; Dr. Rodrigo Urízar E., M.D.2; Dr. John Kaplan, Ph. D. ${ }^{2}$; \\ Dt. Gerald K. Mizejewski, Ph. D.2; Dt. Robert J. Simon, B.S. ${ }^{2}$. \\ Glomerulitis Induced by Experimental Inyection of Inmune \\ Complexes (I.C.) in Rats. The Effects of Amniotic Fluid on IC Clearance.
}

\begin{abstract}
A nomal cleafance of immune complexes (IC), measured as phagocytic index, was observed in C57L/J mice 10 min after one dose of 125 I-IC. Similarly, tissue uptake of radioactivity (percent of dose) was within normal limits when evaluated 15 and $120 \mathrm{~min}$ after injection. Daily dose of IC (for $\$$ days) or pretreatment of mice with amniotic fluid containing alpha-fetoprotein (MAF/AFP) reduced significantly ( $\mathrm{p}<0.01$ ) both phagocytic index and percent of dose at 15 min when only one dose of $\mathrm{K}$ was given.Conversely, at 120 min the percent of dose in liver and kidney was incresed $(p<0.01$ ). In untreated mice the percent of dose was low at 120 min. Pretreatment with AFP-poor MAF had no effect upon clearance or percent of dose. Multiple injections 5 of IC induced mesangial hyperplasia and infiltration with polymorphonuclear and mononuclear leukocy.tes. Distinct granular-nodular fluorescent deposits developed in mesangium and peripheral capillary loops which cortelated well with the election dense material localized in mesangium. Pretreatment with MAF/AFP decreased significantly or prevented these lesions. Pretreatment with AFP - poor - MAF did not modify the inmunohistopathology. These previously unreported effects of MAF/AFP may represent inhibition of the reticuloendothelium. It is postulated that the increased radioactivity detected at 120 min may reflect reduced degradation of IC as opposed to increased uptake.

(Key words: Alpha-fetoprotein. Amniotic fluid. Induced Glomerulitis. Inmune Complexes. Inmunohistopathology. Inmune complex's Clearance. Reticuloendothelial system inhibition by amniatic fluid).
\end{abstract}

En la mayoría de las glomerulonefritis (GN), el mịecanismo desencadenante envuelve la producción de Inmuno-complejos (IC) ${ }^{\mathbf{1 . 7}}$. Ya sea que los IC se formen en la circulación o "in situ" se desarrolla evidencia clínica e histopatológica de GN si la dosis del antígeno, la formación de anticuerpos por el huésped y los procesos que facilitan la localización de antígenos o de IC son adecuados $1-7-9-11 \cdot 13 \cdot 14$

La administración de IC preformados es capaz de desencadenar GN, aunque sus resultados no son tan reproducibles como los obtenidos con inmunización activa en el animal experimental" 7 - 12. La activación del sistema reticuloendotelial (SRE), que fagocita y depura los IC, puede,

1. Departamento de Fisiología y Medicina Intema, Univetsidad de la República, Montevideo Uruguay.

2. New York State Department of Health, Wadsworth Center for Laboratories and Research, Albany, N.Y. 12201, and Departments of Pediatrics and Physiology, Albany Medical College, Albany, N.Y. 12208, U.S.A. en forma efectiva, evitar que éstos se depositen en los tejidos ${ }^{15-18}$. Sin embargo no se ha establecido claramente sj la inhibición del SRE facilita o disminuye el depósito de IC en el riñón ${ }^{1}-19$. 20. Aûn más, la interpretación de la histopatología renal en roedores se complica debido a que estos animales desarrollan espontáneamente lesiones glomerulares con inmunofluorescencia que sugiere un fenómeno mediado por la localización capilar de $\left[\mathrm{C}^{1}-4\right.$ - 21 - 28 .

Los objetivos del presente estudio evalúan, primero: la función del SRE, la inmunohistopatología y ultraestructura renal de ratones $\mathrm{C} 57 \mathrm{~L} / \mathrm{J}$ inyectados con IC preformados. Segundo, el efecto del LA (Líquido Amniótico) que contiene alfafetoproteína (AFP, LA/AFP), una substancia posiblemente inmunosuptesora ${ }^{29}$ sobre la función depuradora de IC del SRE. Por último, se realizaron estudios morfométricos y longitudinales, a través de varias edades, en ratones $\mathrm{C} 57 \mathrm{~L} / \mathrm{J}$ con el objeto de comparar las alteraciones glomerulares espontáneas con aquellas inducidas por Los IC. 


\section{MATERIAL Y METODO}

Se prepararon y purificaron anticuerpos radioactivos anti-seroalbúmina bovina (SAB) desde suero de conejo anti-SAB preparado comercialmente, mediante cromatografía de colum. $n^{30-31}$, obtenjéndose una concentración final de $5.5 \mathrm{mg}$. de proteína/ml. Mediante inmunodi. fusión en agar, se determinó la pureza del anticuerpo de conejo contra $\mathrm{SAB}$, utilizando varios anticuerpos anti-lg de conejo y una solución de $\mathrm{SAB}(40 \mathrm{mg} / \mathrm{ml}$ ). En tọdos los casos se obtuvo una banda única de precipitación.

Se yoduró el anticuerpo anti-SAB usando una modificación del procedimiento de la lactopero. xidasa-glucoxidasa ${ }^{32-33}$ (New England Nuclear, Boston, MA, USA); con este objeto, $200 \mu \mathrm{l}$ de anticuerpo (de conejo) anti-SAB se mezclaron con $2 \mathrm{mCI}$ de $\mathrm{I}^{125}(20 \mu \mathrm{l}, \mathrm{pH} 8-10)$ y $50 \mu \mathrm{l}$ de una solución al $1 \%$ (peso/vol) de glucosa BD. E1 producto final demostró $0.4 \mathrm{mg}$ de proteína por ml. Esta solución fue dializada en BPS ( $\mathrm{pH}$ 7.4) y en la solución obtenida se observó una radioactividad de $0.5 \mathrm{mCi} / \mathrm{ml}$ con una actividad específica de $1.25 \mathrm{mCi} / \mathrm{mg}$ de proteina.

La preparación y yoduración de los IC se realizó precipitando un liofilizado de anticuerpo de conejo anti-SAB $(2.2 \mathrm{mg}$. anticuerpo/ml) en equivalencia con antígeno SAB. Los IC así formados se disolvieron agregando un exceso de antígeno correspondiente a 2 veces la cantidad utilizada para producir la precipitación. El pH se redujo a $3.0^{12}$ y los IC disueltos en exceso de antígeno se inyectaron dentro de un período de 24 hrs. A estos IC se les agregó $40 \mu \mathrm{d}$ del anti-SAB marcado con $\mathrm{I}^{125}(0.016 \mathrm{mg}$ proteína y $20 \mathrm{mCi} /$ ratón); la mezcla, se agitó vigorosamente inmediatamente antes de su inyección.

Para la obtención del LA y preparación de un estándar de alfafetoproteina (AFP), se puncionó el saco amniótico de ratones NYS/Nular con embarazo de 15 a 18 dias, y el LA obtenido se procesó como describieran Mizejewski y colaboradores ${ }^{34}$.

La concentración de AFP medida con inmunodifusión radial ${ }^{35}$, fue de $1.0 \mathrm{mg} / \mathrm{ml}$ mientras que la concentración de proteina total usando el procedimiento de Lowry ${ }^{36}$, fue de $2,75 \mathrm{mg} / \mathrm{ml}$. La preparación del estándar de AFP se llevó a cabo usando anticuerpo anti-AFP a través de los procedimientos publicados por Mizejewski y colaboradores ${ }^{37-38}$. La concentración de AFP del estándar (inmunodifusión y electroforesis) fue de $100 \mu \mathrm{g} / \mathrm{ml}$. La pureza de la AFP usada para la preparación del anticuerpo fue documentada por medio de electroforesis en gel de poliacrila. mida $^{39}$, mientras que la monoespecificidad del estándar de AFP se controló verificando su reactividad con el antígeno purificado y con anticuerpo monoespecifico de conejo anti transfertina, IgG y albúmina de ratón. Se obtuvo LA_con cantidades minimas de AFP pasándolo a través de una columna cromatográfica que contenía estradiol $\left(\mathrm{E}_{2}\right.$, separación de la $\mathrm{A} \overrightarrow{\mathrm{F} P}$ porcromatografía de afinidad $)^{40}$.

Estudio experimental A. Evaluación del efecto del LA sobre la depuración (clearance) y distribu. ción tisular de IC. Con este objeto se utilizaron ratones machos de 2 meses de edad ( 25 a $30 \mathrm{gr}$ ), alimentados con dieta estándar de animal de laboratorio y libre acceso a agua.

La depuración de IC-I $\mathrm{I}^{\mathbf{1 2 5}}$ se estudió en 79 ratones separados en 5 grupos: Treinta y tres ratones se inyectaron por via endovenosa con 0,1 $\mathrm{ml}$, de una solución de IC ( $2 \times$ exceso de antígeno) conteniendo $20 \mu \mathrm{Cl}$ de $\mathrm{I}^{125} ; 17 \mathrm{de}$ ellos recibieron sólo esta inyección; los 16 restantes recibieron IC no marcados - una inyección diaria- por 4 dias, mientras que la quinta inyección fue de IC- $\mathrm{I}^{125}$ en las dosis mencionadas antes (Grupos $\mathrm{IC}_{1}$ sin LA y Grupos $\mathrm{IC}_{5} \sin \mathrm{LA}$ ). Otros 31 ratones fueron inyectados por vía intra. peritoneal con $10 \mu \mathrm{l}$ de LA 3 veces por semana durante 4 semanas ( 12 inyecciones de LA); aproximadamente una hora después de la última inyección, 15 rátones recibieron una dosis de IC.) 125 (Grupo IC, + LA) en tantó que los 16 restantes fueron inyectados diariamente con una dosis de IC no marcados por 4 dias y al quinto día se le inyectó con IC-I $\mathrm{I}^{125}$ (Grupo $\mathrm{IC}_{5}+\mathrm{LA}$ ). Finalmente, el último grupo de 15 ratones fue tratado durante cuatro semanas con LA sin AFP $y$ se les dio al final de este periodo una inyección de [C-I 25 (Grupo ]C 1 + E $2 /$ LA) 2, 2, 4, 8 y 10 min. después de la dosis de $\mathrm{IC}^{-\mathrm{l}^{125}}$ se extrajeron $10 \mu l$ de sangre. La mitad de los animales de cada grupo fue sacrificada a los 15 minutos y la otra mitad a los 120 minutos. El hígado, el bazo, los pulmones y los riñones fueron extraídos, secados y pesados; una muestra de sangre fue obtenida y también fue pesada. La radioactividad de la sangre, el estándar y los tejidos se midió en un con. tador analítico. La evaluación de la depuración de los IC-I ${ }^{125}$ se realizó en las muestras obtenjdas en los primeros 10 minutos mientras que e] conteo de los tejidos se hizo a $\operatorname{los} 15$ y 120 minutos. La dosis de radioactividad se calculó a partir de los estandar. La pendiente e intersección y el coeficiente de correlación de la curva de depuración se calculó mediante análisis regresivo del logaritmo de la radioactividad sanguínea versus tiempo, la constante $\mathrm{k}\left(\mathrm{k}=\mathrm{min}^{-1}\right)$ o indice fagocitico y la intersección extrapolada al tiempo $O(t=0)$ como $10^{-5}$ unidades de radioactividad por minuto por $\mathrm{ml}$ de sangre. La radioactividad 
de los tejidos se calculó pesando 2 muestras del órgano en particular obteniéndose la radioactividad por gramo tejido. Este resultado fue multiplicado por el peso del órgano para indicar la radioactividad total del organo. Con este valor se calculó, el porcentaje de la dosis inyectada, proporción que se usó para expresar los resultados a los 15 y a los $120 \mathrm{~min}$.

Detección de IC no marcados: 42 ratones C57L/J (machos) de 2 meses de edad se trataron con IC no yodurados, con LA y con LA $\sin$ AFP como se detalló antes. Los grupos $I_{C_{1}} \sin \mathbf{L A}$, con LA y con LA sin AFP contenian 7 animales cada uno, mientras que los grupos $\mathrm{IC}_{3}$ sin LA y con LA tenían 11 a 9 ratones respectivamente. Los animales fueron sacrificados 2 hrs. después de la última inyección de IC obteniéndose mues. tras de rifión para su estudio por microscopía de luz (ML), microscopia de fluorescencia (MIF) y microscopia electrónica (ME).

La detección de IC circulantes se realizó mediante el uso de las pruebas de precipitación con polietilenglicol (PEG) y de agregación plaquetaria (AP).

Para la precipitación con PEG (peso molecular 6000) este se usó en una concentración final de $2.5 \% 41$. Las inmunoglobulinas $\mathrm{G}$ y $\mathrm{M}$ y $\mathrm{C}_{3}$ del ratón fueron evaluadas en duplicado en el precipitado inducido por el PEG, usando anticuerpos monoespecíficos preparados en conejos. Los resultados se consideraron positivos (indicando presumiblemente la presencia de IC circulantes) cuando se detectó una línea única de precipitación por lo menos en 2 de las tres proteínas investigadas. Como control positivo se utilizaron una solución de IC preparados en exceso de antígeno y una solución de IgG humana agregada mediante calor. Los controles negativos fueron muestras de suero humano y murino normales.

Agregación Plaquetaria ${ }^{42}$ : Se prepararon suspensiones de plaquetas humanas utilizando muestras de $25 \mathrm{ml}$ de sangre (anticoagulada con ACD) extraida de dadores normales. La concentración plaquetaria final fue de $2 \times 10^{5}$ y la suspensión se empleó dentro de $6 \mathrm{hrs}$. de su preparación. Las muestras séricas a evaluar $(100 \mu l)$ fueron diluidas 1:4 y sucesivamente hasta 1:1.024 e incubadas en placas de microtitulación. Se mantuvieron por 12 hrs. a $40 \mathrm{C}$ antes de ser leidas. La presencia de una capa uniforme de plaquetas sedimentadas en dilución de 1:16 o mayor se consideró positiva, mientras que los "Rouleau" o un botón claramente definido correspondió a un resultado negativo. Como control positivo se usó IgG humana agregada (que produce precipitación en diluciones hasta 1:1.024); como control negativo una solución de plaquetas en tampón fosfato/glucosa.
En el momento de sacrificar a los animales se realizó un examen urinario de rutina (Dipstix) y microscópico en muestras obtenidas directamente de la rejiga.

Para los estudios morfológicos con Microscopía de luz (ML) $(n=41)$, las muestras de tejido renal se procesaron y estudiaron semicuantitativamente como se publicara antes ${ }^{43}$. La Glomeruloesclerosis y las lesiones de la pared capilar, de la membrana basal capilar y del mesangio se clasificaron como ausentes (0); mínimas (I+); moderadas $(2+)$ y severas $(3+)$. Las lesiones tisulares que comprome tían algunos o $75 \%$ o más de los glomérulos se definieron como focales o difusas respectivamente, mientras que aquellas que envolvían algunas áreas o $75 \%$ o más del ovillo glomerular se les consideró segmentarias o globales respectivamente. El número de $\mathrm{PMN}$ en los glomérulos se evaluó en forma semicuantitativa. La microscopía electrónica $(\mathrm{ME})(\mathrm{n}=20) \mathrm{se}$ realizó de acuerdo con procedimientos rutinatios previamente publicados ${ }^{4-44}$ en 3 a 4 glomérulos de 5 a 6 ratones de cada grupo. Microscopía de inmu nofluorescencia (MIF) $(n=39)$ : Usando el procedimiento directo, se detectó IgG, IgM e IgA de ratón; para demostrar $C_{3}$, se empleó la técnica indirecta (anticuerpo monoespecífico preparado en conejo y anticuerpo anti-lg de conejo preparado en cabra); la especificidad del antisuero se determinó mediante inmunodifusión doble, inmunoelectroforesis y pruebas de inhibición. La intensidad $y$ distribución de la fluorescencia se evaluaron como se detalló antes.

Los resultados de este estudio y los del estudio B, fueron sometidos a análisis estadístico. Los resultados individuales y por grupos etarios se compararon mediante análisis de $X^{2}{ }^{45}$. Los promedios $y$ las desviaciones estándas del espesor de la membrana basal fueron calculados para animales individuales y para los distintos grupos usando pruebas $t^{46}$.

Estudio experimental $B$ : La evaluación de la glomerulopatía espontánea de la cepa C57L/J se realizó de manera funcional y morfométrica en 67 ratones (machos) que se distribuyeron en la siguiente forma: Grupo 1, de 1 a 4 meses de edad $(n=7)$; Grupo 2, 6 a 9 meses $(n=26)$; Grupo 3, 12 a $15(n=11)$; y Grupo 4,18 a 24 meses de edad $(\mathbf{n}=13)$. Los ratones anestesiados fueron sometidos a extracción sanguinea total: ambos riñones fueron extirpados $y$ las muestras procesadas cono se señaló antes para ML, MIF, y ME. El grosor de la membrana basal se midió de acuerdo con procedimientos publicados anteriormente, en 5 a 10 capilares glomerulares de 4 a 8 ratones de cada grupo 44 . La creatinina sérica se evaluó en un total de 40 muestras de los 4 grupos usando autoanalizador. 


\section{RESULTADOS}

Estudio Experimental A: Depuración de IC-1 ${ }^{125}$ (Tabla 1): los animales que recibieron una inyección de $\mathrm{IC}^{1} \mathrm{I}^{125}\left(\mathrm{IC}_{1} \sin \mathrm{LA}\right)$ mostraron una depuración de 0.012 imin-1 correspondiente a un tiempo medio de aproximadamente 25 min. Los ratones inyectados con LA $\left(\mathrm{IC}_{1}+\mathrm{LA}\right)$ presentaron una depuración significativamente reducida. La depuración se mantuvo nomal cuando el líquido amniótico (LA) usado para tratamiento previo a la inyección de $\mathrm{IC} \cdot \mathrm{I}^{125}$ contenía cantidades mínimas de AFP $\left(\mathrm{IC}_{1}+\mathrm{E}_{2} /\right.$ LA). Los ratones inyectados con 5 dosis de IC sin LA (IC $C_{s}$ sin LA) o con AFP ( $\left.\mathrm{C}_{\xi} \times \mathrm{LA}\right)$ presentaron una reducción semejante de la depuración de IC-I ${ }^{125}$ de la sangre.

La radioactividad de los órganos (Tabla 1) estaba considerablemente disminuida a los 15 min. en el grupo tratado con $\mathrm{LA}\left(\mathrm{IC}_{1}+\mathrm{LA}\right)$ pero no fue modificada por el tratamiento con LA sin $\mathrm{AFP}\left(\mathrm{IC}_{1}+\mathrm{E}_{2} / \mathrm{LA}\right)$. En contraste con estos resultados, a los $120 \mathrm{~min}$, el grupo que recibió LA, demostró un aumento considerable en el porcentaje de radioactividad. En los grupos de control $\left(\mathrm{IC}_{1} \sin \mathrm{LA}\right.$ y $\mathrm{IC}_{1}+\mathrm{E}_{2} / \mathrm{LA}$ ) con radioactividad renal alta (y depuración normal) a los $15 \mathrm{~min}$, se observó una notable disminución a los $120 \mathrm{~min}$, mientras que aquellos con reducción de la depuración $\left(\mathrm{IC}_{1}+\sin \mathrm{LA}\right.$ y $\left.\mathrm{IC}_{5}+\mathrm{LA}\right)$ y radioactividad baja en los rifrones hubo por una elevación significativa de ésta a los $120 \mathrm{~min}$. El coeficiente de correlación entre la depuración de IC-I ${ }^{125}$ y la localización de $\mathrm{IC}$ en el riñón, fue de alta significación estadistica $(r=0.92, p<0.01)$. Esta correlación fue negativa $(\mathrm{r}=0.86, \mathrm{p}<0.01)$ cuando la depuración se comparó con la localización a los $120 \mathrm{~min}$.

La creatinina sérica se mantuvo normal en todos los grupos, pero la excreción de eritrocitos aumentó considerablemente en ratones, inyectados con 5 dosis de IC. Las pruebas de precipitación de $\mathrm{PEG}$ y de $\mathrm{AP}$ en grupos $\mathrm{IC}_{1}$ sin LA, IC 5 sin LA y IC 5 + LA revelaron IC circulantes o substancias semejantes a ellos en cant $\mathrm{A}$. dades significativas, sin embargo los titulos de AP fueron estadísticamente mayores en ratones del grupo $\mathrm{IC}_{5} \sin \mathrm{LA}$ que en aquellos que recibieron LA ( $p<0.01$, resultados no incluídos).

En Ja Microscopía de luz (41 ratones, 2 meses de edad, Tabla 2, Figura 1): Una inyección de IC no marcados (Grupo $I C_{1}$ sin $L A$ ), produjo expansión de la matriz mesángica, proliferación segmentaria del mesangio con 1 ó 2 PMN (y MN ocasionales) visibles en el glomérulo, sin edema o aumento del tamaño glonerular. Cinco inyec. ciones de IC (Grupo IC 5 sin LA) indujeron una mesangiopatia caracterizada por aumento de la matriz e hipercelularidad. Los PMN aumentaron considerablemente de 5 a 8 y ocasionalmente hasta 12 leucocitos por glomérulo; los MN aumentaron pero en menor escala que los anterio. res; los glomérulos se veian edematizados y aumentados de tamaño. Los ratones tratados previamente con LA antes de la dosis de IC $\left(\mathrm{IC}_{1}+\mathrm{LA}\right.$ y $\left.\mathrm{IC}_{5}+\mathrm{LA}\right)$ mostraron lesiones mínimas o ausencia de ellas.

En la microscopía electrónica (20 ratones, 2 meses de edad, Tabla 2, Figura 1) se confirmaron los hallazgos histológicos, encontrándose ausen.

Tabla 1.

Efecto de Ia alfafetoproteína sobre la depuración vascular (Indice Fagocítico) y la localización en Hígado y Riñón de los inmuno complejos inyectados (recuento por Min/Mg y porcentaje de la dosis \pm error estándar' (Estudio A)

\begin{tabular}{|c|c|c|c|c|c|c|c|c|c|c|c|c|c|}
\hline \multirow{3}{*}{ GRUPO } & \multirow{3}{*}{$\begin{array}{c}\text { Indice } \\
\text { Fagocítdco } \\
\text { K (mio)- } 1 \\
\text { (+ES) }\end{array}$} & \multirow[b]{3}{*}{ o } & \multicolumn{5}{|c|}{ RINAONES } & \multirow[b]{3}{*}{ n } & \multicolumn{5}{|c|}{ HIGADO } \\
\hline & & & \multicolumn{2}{|c|}{$\longrightarrow \quad 1 \overrightarrow{5} \mathrm{mtr}$} & & \multicolumn{2}{|c|}{$120 \mathrm{~min}}$. & & \multicolumn{2}{|c|}{15 min. } & & \multicolumn{2}{|c|}{$120 \mathrm{~min}$} \\
\hline & & & $\begin{array}{c}c \rho m / m g \\
(+E S)\end{array}$ & $\begin{array}{l}\text { \%dosis } \\
\text { (+ES) }\end{array}$ & n & $\begin{array}{c}c p m / m g \\
(+E S)\end{array}$ & $\begin{array}{l}\text { \%odosis } \\
\text { (+ES) }\end{array}$ & & $\begin{array}{c}\text { cpmimg } \\
\text { t+ESi }\end{array}$ & $\begin{array}{l}\text { \% dosig } \\
\text { (+ES) }\end{array}$ & n & $\begin{array}{c}\text { cpnijmg } \\
(-E S)\end{array}$ & $\begin{array}{l}\text { Tdosis } \\
\text { I+ESI }\end{array}$ \\
\hline$I_{1}(\sin I A)$ & $\begin{array}{c}0.0121 \\
(0.0027)\end{array}$ & 8 & $\begin{array}{c}2.6 \\
(0.3)\end{array}$ & $\begin{array}{c}3.3 \\
(0.1)\end{array}$ & 9 & $\begin{array}{l}273 \\
(14)\end{array}$ & $\begin{array}{c}2.6 \\
(0.5)\end{array}$ & 8 & $\begin{array}{c}4.51 \\
(0.13)\end{array}$ & $\begin{array}{l}22.3 \\
10.81\end{array}$ & 9 & $\begin{array}{l}248 \\
(9)\end{array}$ & $\begin{array}{c}8.3 \\
(0.8)\end{array}$ \\
\hline $\mathrm{IC}_{\mathbf{f}}+\mathrm{L}_{\mathbf{A}} \mathrm{FR}$ & $\begin{array}{c}0.007) \\
(0.0012)\end{array}$ & 8 & $\begin{array}{c}2.0 \\
(0.2)\end{array}$ & $\begin{array}{c}2.7 \\
(0.3)\end{array}$ & ? & $\begin{array}{l}291 \\
(35)\end{array}$ & $\begin{array}{c}3.7 \\
(1.4)\end{array}$ & 8 & $\begin{array}{c}3.78 \\
(0.16)\end{array}$ & $\begin{array}{l}21.4 \\
(0.7)\end{array}$ & 7 & $\begin{array}{l}230 \\
(28)\end{array}$ & $\begin{array}{c}9.8 \\
(3.1)\end{array}$ \\
\hline$\left[C_{1}+E_{2} / L_{-A}\right.$ & $\begin{array}{c}0.0167 \\
(0.0020)\end{array}$ & 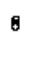 & $\begin{array}{c}2.2 \\
(0.1)\end{array}$ & $\begin{array}{c}3.5 \\
(0.1)\end{array}$ & 7 & $\begin{array}{l}165 \\
(14)\end{array}$ & $\begin{array}{c}2.4 \\
(0.2)\end{array}$ & $\mathbf{0}$ & $\begin{array}{c}3.21 \\
(0.13)\end{array}$ & $\begin{array}{l}18.9 \\
(0.2)\end{array}$ & $?$ & $\begin{array}{l}135 \\
(9)\end{array}$ & $\begin{array}{c}7.1 \\
(0.3)\end{array}$ \\
\hline$t C_{5}(\sin [-A)$ & $\begin{array}{c}0.0082 \\
(0.0014)\end{array}$ & $\mathbf{8}$ & $\begin{array}{c}1.3 \\
(0.08)\end{array}$ & $\begin{array}{c}2.5 \\
(0.16)\end{array}$ & 8 & $\begin{array}{l}231 \\
(32)\end{array}$ & $\begin{array}{c}2.9 \\
11.05)\end{array}$ & 8 & $\begin{array}{c}3.39 \\
(0.24)\end{array}$ & $\begin{array}{c}24.7 \\
(2.09)\end{array}$ & 8 & $\begin{array}{l}298 \\
(43)\end{array}$ & $\begin{array}{c}14.7 \\
(5.80)\end{array}$ \\
\hline $\mathrm{IC}_{\mathrm{O}}+\mathrm{LA}$ & $\begin{array}{c}\text { D.0080 } \\
(0.0009)\end{array}$ & 8 & $\begin{array}{c}1.3 \\
(0.2)\end{array}$ & $\begin{array}{c}2.5 \\
(0.3)\end{array}$ & 8 & $\begin{array}{l}326 \\
\text { (1B) }\end{array}$ & $\begin{array}{c}3.7 \\
(0.7)\end{array}$ & 8 & $\begin{array}{c}3.34 \\
(0.30)\end{array}$ & $\begin{array}{c}21.0 \\
2.1\end{array}$ & 0 & $\begin{array}{l}367 \\
75\end{array}$ & $\begin{array}{l}14.5 \\
(2.5)\end{array}$ \\
\hline
\end{tabular}

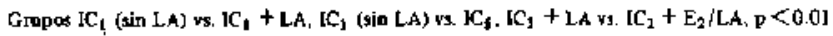

Gropos $K_{1}$ (ain $L A$ ) PQ. $I C_{1}+E_{2} / L A$ y $l C_{5}$ (gin $\left.L A\right)$ rs. $\left[C_{\$}+L A, p>0.0 s\right.$ 
cia de lesiones o lesiones mínimas en el Grupo $\mathrm{IC}_{1}$ con LA. Los ratones que recibieron 5 inyecciones de IC $\left(\mathrm{IC}_{5} \sin \mathrm{LA}\right)$ presentaron depósitos densos focales y segmentarjos en el mesangio y el subendotelio, con la expansión de la matriz y la proliferación celular antes descrita. PMN y MN fueron fácilmente identificados, observándose ocasionalmente trombos con fibrina y plaquetas en los lúmenes capilares. Los ratones tratados previamente con $\mathrm{LA}\left(\mathrm{IC}_{5}+\mathrm{LA}\right)$ no desarrollaron lesiones significativas.

En el examen con microscopía de inmuno. fluorescencia ( 39 ratones, 2 meses de edad, Tabla 2, Figura 1) todos los grupos presentaron depósitos mesangiales espontáneos de IgG e IgM de intensidad mínima $(1+/ 2+)$. La fluorescencia indirecta demostró depósitos de IgG de conejo anti $S A B$ en mesangio y capilares periféricos en los grupos $\mathrm{IC}_{1} \sin \mathrm{LA}$ y $\mathrm{IC}_{1}+\mathrm{E}_{2} / \mathrm{LA}$, mientras que los depósitos en esas localizaciones eran intensamente positivos en el grupo $\mathrm{IC}_{5} \sin \mathrm{LA}$. El tratamiento con LA redujo considerablemente la intensidad $y$ extensión de la fluorescencia (Grupos $\mathrm{IC}_{1}$ LA y $\mathrm{IC}_{5}+\mathrm{LA}$ ). El Antígeno o SAB (seroalbúmina bovina) no se detectó en ninguno de los grupos.

Estudio Experimental B: La creatinina sérica se mantuvo normal $(0.5$ a $0.6 \mathrm{mg} / \mathrm{ml})$ en todos los grupos. El examen de orina de ratones jóvenes ( 2 meses) demostró proteinuria mínima (trazas a 1+) mientras que en ratones de 12 a 24 meses de edad se detectó consistentemente en concentra- ciones de $2+$ o más. La prueba de precipitación con PEG fue positiva en 4 de 10 y 16 de 19 ratones en los grupos 1 y 3 respectivanente; el contenido de IgG, IgM, y $\mathrm{C}_{3}$ fue mayor $(p<0.01$ ) en el grupo 3 (resultados no incluídos). La prueba de AP fue positiva en todos los ratones, sin diferencias significativas en ninguno de los grupos mayores comparados con animales jóvenes (Grupo 1).

En la microscopía de luz (67 ratones, Tabla 3 , Figura 2) el Grupo 1 (1-4 meses) no presentó lesiones o mostró sólo cambios minimos (hiperplasia focal segmentaria cerca de ó en el hilio glomerular. En el grupo 2 (6-9 meses) se comprobo hiperplasia mesangica y algunas protuberancias argirofílicas en las membranas basales. En el grupo 3 (12-15 meses) se observó proliferación segmentaria del endotelio y epitelio, hiperplasia mesángica, hialinización y aumento del grosor de la pared capilar (severidad $2+$ ). La membrari basal glomerular (mbg) aparecía estructuralmente normal. Algunos focos de infiltración mononu. clear intersticial o periglomerular se observaron con cierta frecuencia. El Grupo 4 (18-24 meses) demostró aumento de estas lesiones $(3+)$ con expansión periférica del mesangio formando contornos capilares dobles. La hialinización fue mayor pero siguió siendo focal $y$ segmentaria. Algunas paredes capilares aparecían engrosadas y arrugadas mientras que las protuberancias de ta mbg aumentaron considerablemente en número $y$ tamaño.

Tabla 2.

Resultados histopatológicos en ratones $\mathrm{C57L/J}$ inyectados con jnmunocomplejos SAB-ANTI SAB, con y sin tratamiento con AFP (Estudio A)

\begin{tabular}{|c|c|c|c|c|c|c|c|c|c|c|c|}
\hline \multirow[b]{2}{*}{ Grupo } & \multicolumn{4}{|c|}{ MICROSCOPIA DE LUIZ } & \multicolumn{4}{|c|}{ LLT RALSTKUCTURA } & \multicolumn{3}{|c|}{ "INMUNOFLLORESCENCIA } \\
\hline & n & $\begin{array}{l}\text { Pered } \\
\text { Capilat }\end{array}$ & Mctengio & $\begin{array}{l}\text { PUN! } \\
\text { GLOM }\end{array}$ & $\mathbf{n}$ & Melangly & Doposites & PMN & n & $\begin{array}{l}\text { Tode Coneje } \\
\text { (andisers de } \\
\text { sabra) }\end{array}$ & $\begin{array}{l}\text { BSA } \\
\text { (an 1,suero } \\
\text { de conejo) }\end{array}$ \\
\hline $\mathrm{IC}_{3}\left(\mathrm{o} \mathrm{LA}_{\mathrm{A}}^{* *}\right)$ & 3 & 0 & $\begin{array}{l}\text { j }+ \text { expansiósi } \\
\text { tocal y segmen- } \\
\text { taria de la } \\
\text { marsiz }\end{array}$ & 2 & 4 & $\begin{array}{l}\text { Exgansido } \\
\text { seymentafla } \\
\text { y focidl keye } \\
\text { endoteliaj }\end{array}$ & $\begin{array}{l}\text { Ocasionat } \\
\text { monte en } \\
\text { metsingio y }\end{array}$ & $\begin{array}{l}\text { Ocasio- } \\
\text { cal }\end{array}$ & 7 & $\begin{array}{l}2-\text { Fotsl y } \\
\text { cemeotario } \\
\text { co mescogio } \\
\text { y petiferiz }\end{array}$ & Nrgalivo \\
\hline$I C_{1}-L a$ & $i$ & $\begin{array}{c}0 \\
\text { (disten- } \\
\text { dixla) }\end{array}$ & $\begin{array}{l}0 \mathrm{dj}+. \\
\text { Exparisión } \\
\text { seqmentads } \\
\text { ocasional }\end{array}$ & ì 1 & 4 & 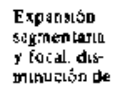 & $\begin{array}{l}\text { Ousional. } \\
\text { ronte en } \\
\text { mesangio y } \\
\text { subchdolelisl }\end{array}$ & $\begin{array}{l}\text { Oussio- } \\
\text { nal }\end{array}$ & 8 & $\begin{array}{l}\text { I a } 2 \\
\text { mesangial } \\
\text { Jiruso }\end{array}$ & Negalivo \\
\hline$\left[C_{1}+E_{J} / L A\right.$ & $?$ & 0 & $\begin{array}{l}1+\text { Enpansión } \\
\text { sogmentaria } \\
\text { [ocel }\end{array}$ & 021 & 4 & Leve, acal & $\begin{array}{l}\text { Ocssional } \\
\text { mesangial }\end{array}$ & $\begin{array}{l}\text { Ocasio- } \\
\text { nal }\end{array}$ & s & $\begin{array}{l}\text { Negrativo a } \\
1 \rightarrow \text { merangal } \\
\text { sequentanio }\end{array}$ & Negativo \\
\hline$\left[C_{\uparrow}\right.$ iO L $\mathrm{R}$ ) & 11 & $\begin{array}{l}\text { 1+az+ } \\
\text { disien- } \\
\text { diday } \\
\text { lumen } \\
\text { disminuido }\end{array}$ & $\begin{array}{l}2 \text { + expandido } \\
\text { más difusw, } \\
\text { prolferackin } \\
\text { segner,taria }\end{array}$ & $\begin{array}{l}5.0 \mathrm{ca} \\
\text { sionst } \\
\text { hasto } 12\end{array}$ & 4 & $\begin{array}{l}\text { Expandido } \\
\text { celulart } \\
\text { dad mono } \\
\text { nuiveles }\end{array}$ & $\begin{array}{l}\text { Frecipente } \\
\text { mess nigial } \\
\text { ysubendo- } \\
\text { ielial }\end{array}$ & $\begin{array}{l}\text { Елесиел- } \\
\text { te agunos } \\
\text { cosd nótilus }\end{array}$ & 8 & $\begin{array}{l}3 \text { - diruso } \\
\text { messingis } \\
\text { segnenterio } \\
\text { a global }\end{array}$ & Negativo \\
\hline $\mathrm{IC}_{3}+\mathrm{L}_{\mathrm{A}}$ & 9 & $\begin{array}{l}\text { 0a } 1+ \\
\text { distendj- } \\
\text { day } \\
\text { entososada }\end{array}$ & 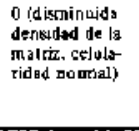 & $11 \times 1$ & 4 & $\begin{array}{l}\text { Nompal o } \\
\text { expinsioncs } \\
\text { segmentalas } \\
\text { infrecuentes } \\
\text { matrly } \\
\text { jeticulal }\end{array}$ & $\begin{array}{l}\text { Sut:radote- } \\
\text { lisl } \\
\text { mesantial }\end{array}$ & $\begin{array}{l}\text { Ocasio- } \\
\text { nel }\end{array}$ & $\theta$ & $\begin{array}{l}\text { Negativo a } \\
\text { 1-mesangial } \\
\text { focel } \\
\text { segimentario }\end{array}$ & Negauva \\
\hline
\end{tabular}

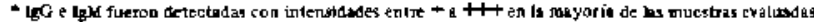

$*$ LA + líquido amniótico. 

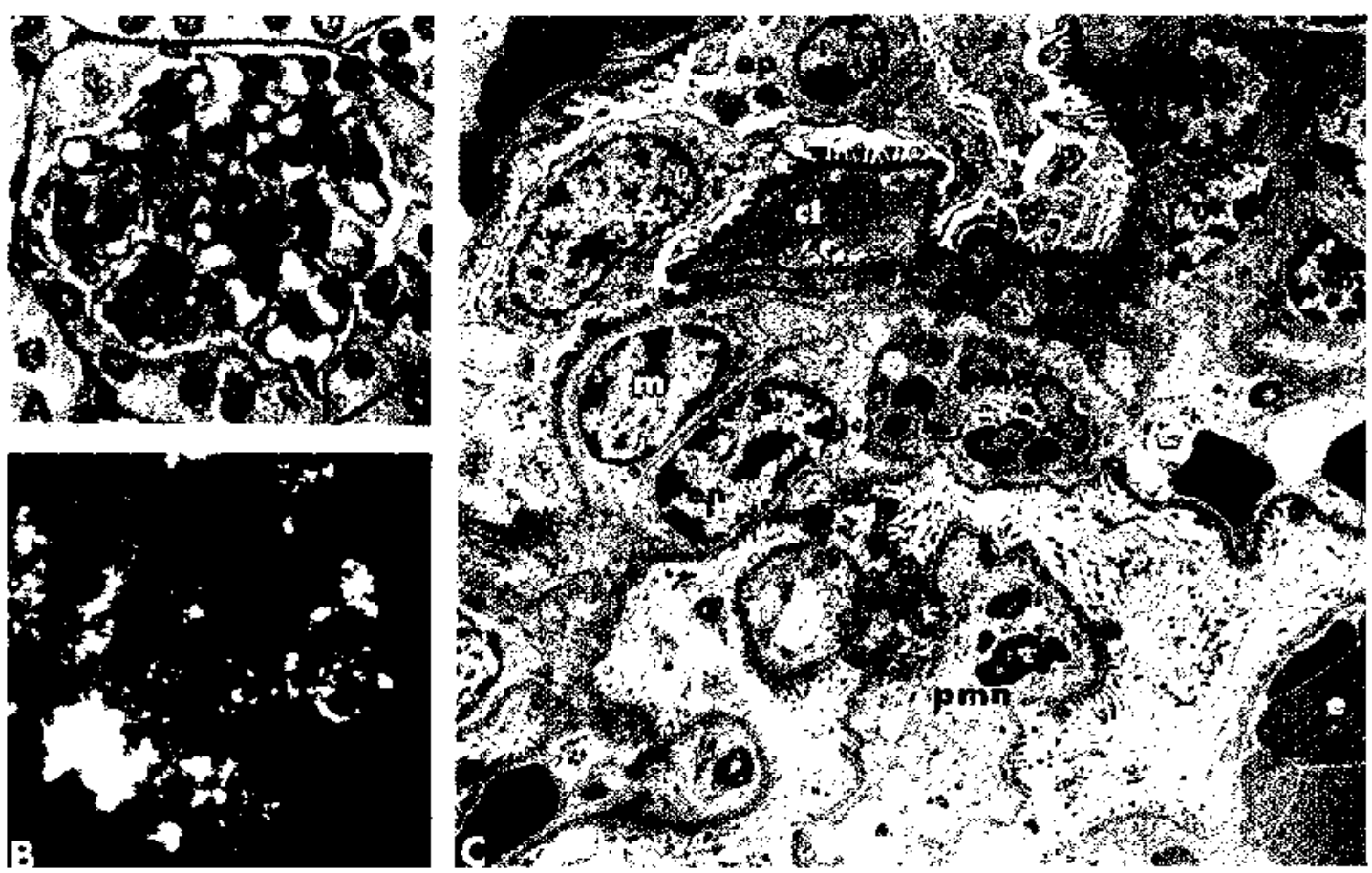

Figura 1. Composición microfotográfica de la microscopía de luz, fuorescencia y electrónica que documenta la

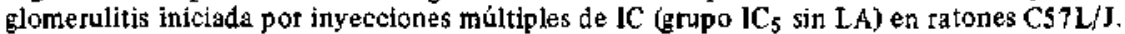

A. Expansión mesángica con aumento celular. Impregnación atgéntica $120 \mathrm{X}$.

B. Depósitos abundantes de anticuerpo anti-IgG de conejo (preparado en cabra), en mesangio e hilio glomerulares, $250 \mathrm{X}$.

C. Hiperplasia mesángica (m), depósitos electrónicos densos (flecha). La pared capilar y los pedicelios aparecen normales. El epitelio (ep) y endotelio (en) están edematizados. Los lúmenes capilares (C1) están abiertos y contienen proteínas y eritrocitos (e). El extremo infetiot derecho muestra una cintura mesángica, leucocitos (pmn y mo) y una célula endotelial (en). Acetato de uranilo y citrato plúmbico, $6000 \mathrm{X}$.

Tabla 3.

Resultados histopatológicos en varios grupos etarios de ratones normales $\mathrm{C57} / \mathrm{L} / \mathrm{J}$ (Estudio $\mathrm{B}$ )

\begin{tabular}{|c|c|c|c|c|c|}
\hline Grupo & $\begin{array}{l}\text { Edad } \\
\text { (theses) }\end{array}$ & $n$ & Microscopia de Luz & LItraestructura & Inmunofluorescencla \\
\hline 1 & $(1-4)$ & 17 & $\begin{array}{l}\text { tr a } 1+\text {. Mesangíal. } \\
\text { focal y segmentaria. } \\
\text { Hiperplasia hiliar. }\end{array}$ & $\begin{array}{l}\text { Oa } 1 \text { t. } \\
\text { Adelgazaniento del } \\
\text { mesangio. Hilio y } \\
\text { mbg normal. }\end{array}$ & $\begin{array}{l}1+\text {. Depósitos granulares } \\
\text { mesangiales de IgG e IgM } \\
\text { de ratón; } C_{3} \text { minimo o } \\
\text { ausente. }\end{array}$ \\
\hline 2 & $(6-9)$ & 26 & $\begin{array}{l}1+\text { a } 2+\text { centuación } \\
\text { de las lesiontes. mbg } \\
\text { permanece normal. } \\
\text { Apatecen protrusiones } \\
\text { argirofílizas. }\end{array}$ & $\begin{array}{l}1+\text { a } 2+\text {. Aumento } \\
\text { de la matriz con pocos } \\
\text { depósitos densos. }\end{array}$ & $\begin{array}{l}2 \quad+\text { Con acentuación } \\
\text { bilias. }\end{array}$ \\
\hline 3 & $(12-15)$ & Il & $\begin{array}{l}2+\text { a } 3 \text { +. Esclerosis } \\
\text { del mesangio. Engrosa- } \\
\text { miento de la pared capilar }\end{array}$ & $\begin{array}{l}2+\text { a } 3+\text {. Escasa } \\
\text { hialinización aumento } \\
\text { de depósitos densos } \\
\text { intramesangial y } \\
\text { subendoteliales. }\end{array}$ & $\begin{array}{l}2+\text { a } 3+\text {. Mayores depósitos } \\
\text { que en algunas áreas son } \\
\text { francarnente nodulares. }\end{array}$ \\
\hline 4 & $\left(\begin{array}{ll}18 & 24\end{array}\right)$ & 13 & $\begin{array}{l}3+\text {. Hialintzación y } \\
\text { expansión periférica } \\
\text { mesangial. Engrosamiento } \\
\text { de la pared capitas y de } \\
\text { mbg. Aumento đe protrusiones } \\
\text { argirofílicas. }\end{array}$ & $\begin{array}{l}3+\text {. Espesamiento } \\
\text { mesangial y esclerosis, } \\
\text { expansiót con capilares } \\
\text { con doble contorno y } \\
\text { protrusiones de la gbm. } \\
\text { aumentados. }\end{array}$ & $\begin{array}{l}3+\text {. Marcadamente mesangial } \\
\text { y a veces depósitos nodulares } \\
\text { granulares en la periferia. }\end{array}$ \\
\hline
\end{tabular}




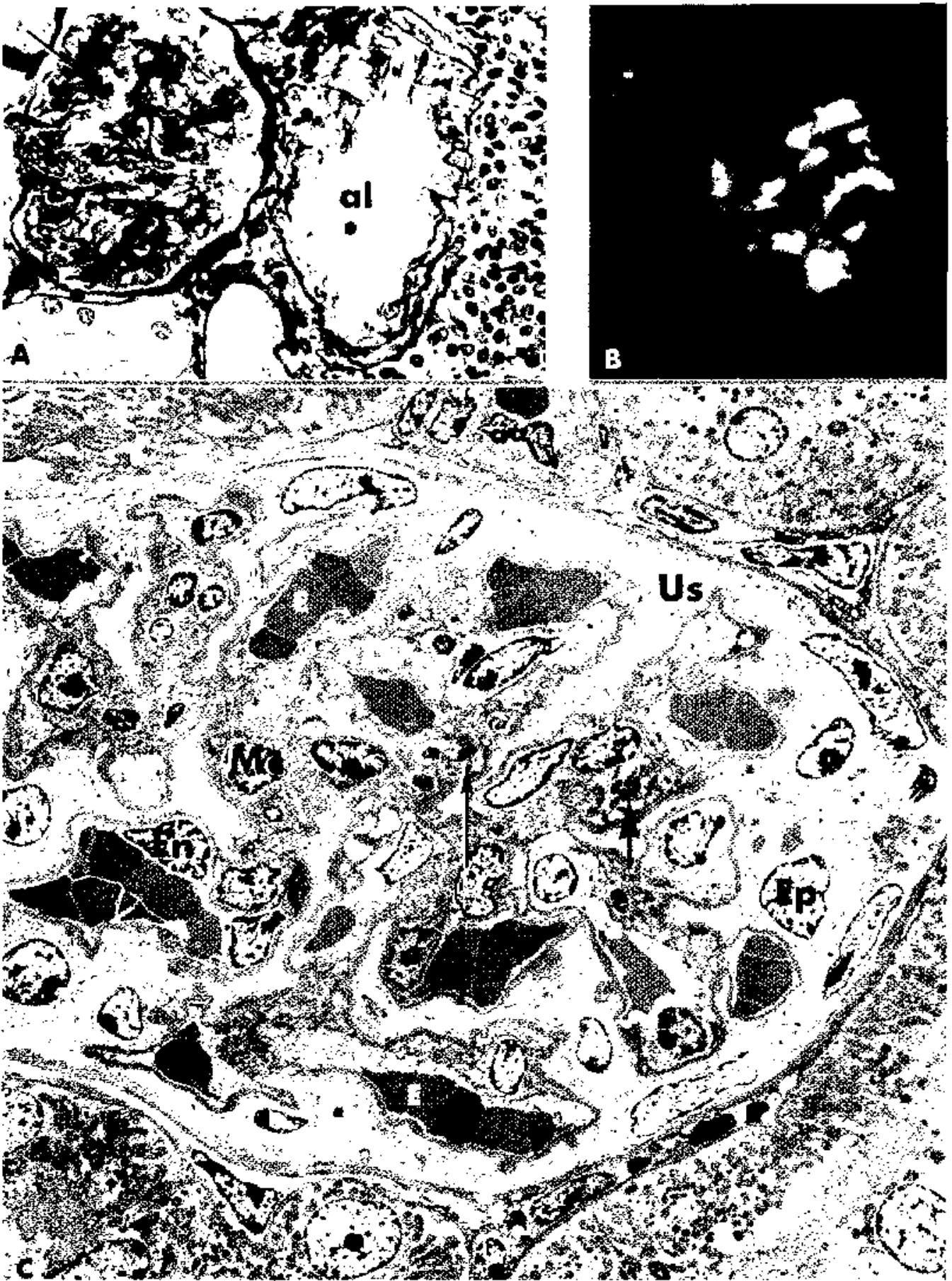

Figura 2. Composición microfotográfica (ML, MIF y ME) que muestra la histopatología glomerular de ratones de edad avanzada (machos) de la cepa C57L/J

A. Esclerosis mesángica, difusa, moderadamente avanzada (flecha) y con acentuación hiliar. Nótese las proyecciones sésiles de la membrana basal (punta de flecha) y el infilteado inflamatorio mononuclear periglomerular (al $=$ lumen arteriolar). Inpịtegnación Afgéntica, $180 \mathrm{X}$.

B. Depósitos mesangiales espontáneos, nodulazes y granulares. Aлticuerpo de conejo, anti-IgM de ratón, $100 \mathrm{X}$

C. Vista panorámica de un glomérulo que destaca la expansión difusa y la esclerosis mesángica. Las flechas indican depósitos densos intramesángicos y endoteliales. $\mathrm{E}=$ eritrocitos; $\mathrm{Ep}=$ epitelio; a $=$ arteriola aferente; Us $=$ espacio urinario.

Nótese los engrosamientos o proyecciones sésiles y pedunculadas de la mbg. Acetato de uranilo y citrato plúmbico, $3000 \mathrm{X}$. 


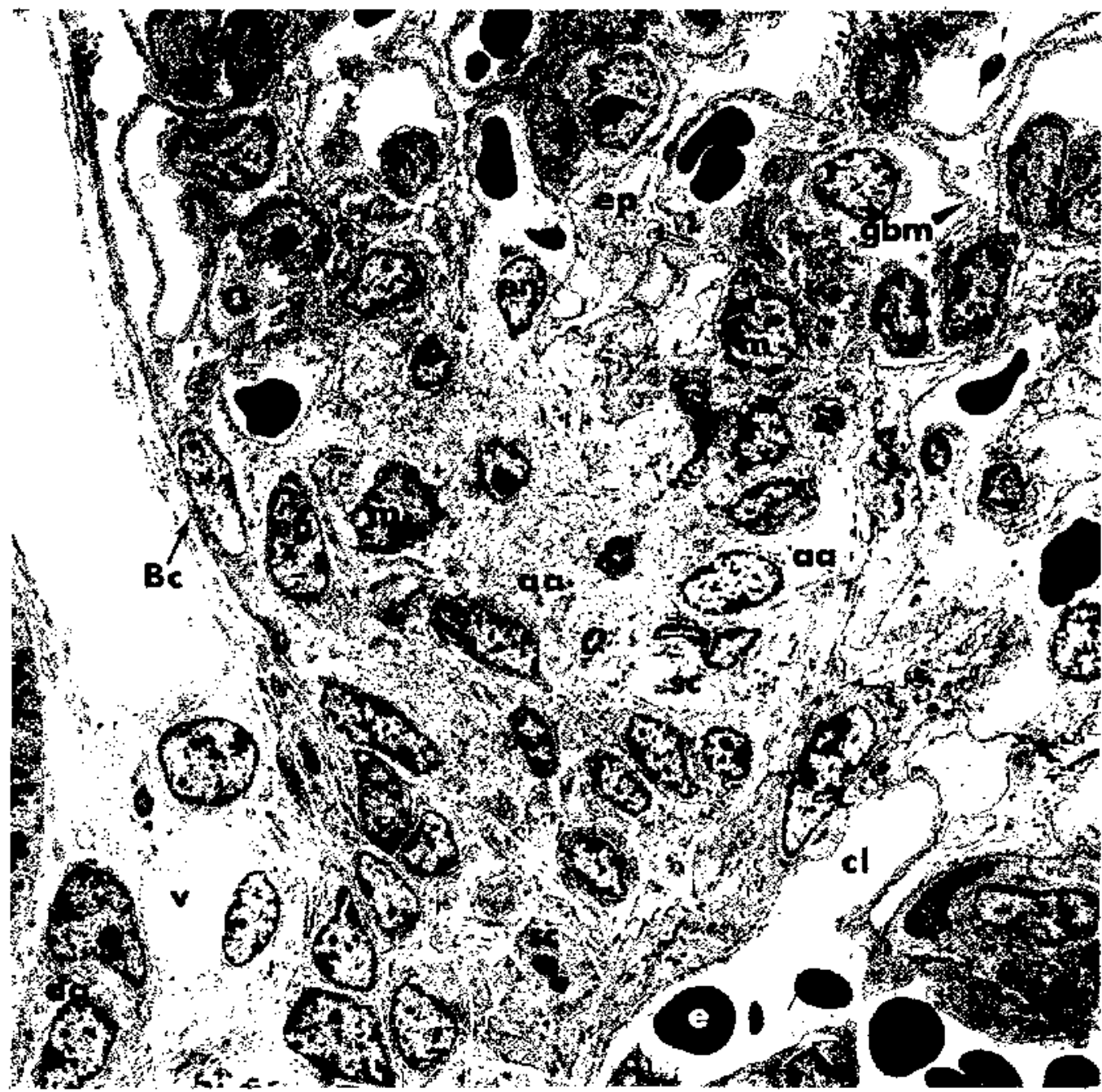

Figura 3. Esta microfotografía electrónica đe bajo aumento (ratón de un mes de edad) destaca la capilarización artetiolar a nivel del hilio glomerular; aa, arteriola aferente; m, mesangio; cl, lumen capilar; sc, músculo liso; $\forall$, vénula; e, eritrocitos; ep, epitelio: gbm, membrana basal glomerular; Bc; cápsula de Bowman. Se destaca claramente en esta ilusiración, la juxtaposición de las células mesángicas y musculares, a medida que se forma el mesangio intraglomerular.

Acetato de ucanilo y citrato plúmbico, $3000 \mathrm{X}$.

En la microscopia electrónica (23 ratones, Tabla 3, Figuras 2 y 3) la estructura hiliar del Grupo 1 aparecía nomal, en el Gnjpo 2 se observaron zonas transicionales entre células musculares y el mesangio extraglomerular con aumento de la cantidad de matriz mesángica. Los Grupos 2, 3 y 4 mostraron expansión progresiva del mesangio, depósitos intramesangiales $y$ subendoteliales segmentarios, granulares o esponjosos. Las protrusiones pedunculadas y sésiles de la mbg estaban aumentadas de tamaño. No se encontraron depósitos subepiteliales y los pedicelios eran normales. En el Grupo 4 la proliferación y fíbrosis mesángica fueron mayo- res con engrosamiento de la pared capilar y de la mbg.

El grosor de la mbg (Tabla 4) fue significativamente mayor $(p<0.01)$ en los Grupos 4 y 3 que en el Grupo 1 .

En ta microscopia de inmunofluorescencia se detectaron depósitos mesangiales de IgG e IgM en todas las edades, particularmente en Grupos 3 y 4. Estos eran generalmente granulares o extendidos y comprometían la mayoría de los gloméru. los y la mayor parte del mesangio. La concen. tración $C_{3}$ fue minima o negativa en todos los Grupos. 
Tabla 4.

Grosor de la membrana basal glomerular medida en varios grupos de edades de ratones normales C57L/J

Membrana Basal Glomerular

\begin{tabular}{ccccc}
\hline Grupo & $\mathrm{n}$ & Grosor (A) & PSDA $^{\mathrm{a}}$ & $\mathrm{p}<0.05$ \\
\hline 1 & 8 & 1526 & 248 & \\
2 & 4 & 1808 & 323 & $(-)$ \\
3 & 6 & 2572 & 522 & $(+)$ \\
4 & 5 & 2810 & 644 & $(+)$ \\
\hline
\end{tabular}

a Desviación estándar acumulada.

\section{DISCUSION}

La depuración de IC en ratones es un fenómeno vatiable, dependiente de la cepa de animales y modulado en gran parte por la actividad del $S^{4} E^{4-49}$. Una depuración acelerada resulta en escasos depósitos tisulares de IC en tanto que la persistencia de estos en la circulación (tránsito prolongado o depuración insuficiente) permite, aparentemente, que grandes cantidades de IC se depositen en los tejidos. Dosis altas de IC o inyecciones múltiples de cantidades relativamen. te pequeñas saturan o bloquean el SRE y deprimen la depuración de IC $^{50.53}$. Los estudios que presentamos están de acuerdo con los trabajos recién mencionados al demostrar la relación inversa que existe entre la depuración de IC y su Jocalización estudiada a las 2 hrs de inyectados. $\mathrm{Sin}$ embargo nuestros resultados están en desacuerdo con los anteriores cuando la evaluación se realiza a los 15 minutos, ya que indican una relación directa caracterizada por una disminución significativa de la depuración de $\mathrm{IC}$ y de la radioacțividad de los tejidos, particularmente del rinón. Nuestros resultados sugieren que el aumento de la radioactividad en los tejidos en presencia de una depuración subnormal de IC se debe posiblemente a una falla del catabolismo local de los IC

Otro hallazgo importante del presente estudio indica que el tratamiento con LA previo a la administración de IC $\cdot \mathrm{I}^{125}$, reduce considerablemente la depuración de IC, lo que no ocurre cuando se ha extraído toda o la mayor parte de la AFP contenida en el LA. De este modo, debido a una destrucción local reducida, los IC permanecen intactos en el rin̄ón permitiendo detectar más tarde cantidades mayores de radioactividad. Es posible por to tanto que los aumentos de radioactividad (IC) detectados en otros trabajos pudieran explicarse a través de este fenómeno.
Hasta ahora no se conocen claramente los mecanismos por los cuales los IC se depositan en las estructuras glomerulares y como los glomérulos se deshacen de estos elementos proinflamatorios. Las ólujas mesángicas consideradas como parte del SRE podrian participar activamente en la degradación de IC o de otras parículas ${ }^{9}$. Existe información reciente que sugiere que los IC se catabolizarían a través de procesos fagociticos no relacionados con las células mesángicas $19 \cdot 54$. 57. La fagocitosis de IC por leucocitos MN localizados en el mesangio ha sido demostrada por varios autores ${ }^{5-58}$, lo que respaldaría nues. tro hallazgo de PMN y MN circulantes ubicados en el glomérulo en ratones inyectados con dosis múltiples de IC. Estos hallazgos histopatológicos eran mínimos o estaban ausentes en ratones tratados con LA antes de la inyección de IC. A pesar de los extensos trabajos de Murgita ${ }^{50.62}$ y de Tomasi ${ }^{29}$ - $63 \cdot 64$ acerca de la posible influencia del LA/AFP sobre el sistema inmunitario, poco se sabe de la influencia que estas substancias podrian tener en la función del SRE. Los resultados presentados sugieren que el SRE es inhibido por el LA/AFP y esta inhibición se manifestaría por una depresión de la depuración $y$ del catabolismo de IC. Es posible que AFP en cierto modo module la respuesta inmunológica, tal vez en la actividad macrofágica como lo han sugerido trabajos preliminares publicados recientemente ${ }^{65}$. Desde un punto de vista morfológico, la mesangeítis proliferativa, con inmunofluorescencia positiva y los depósitos electrónicos densos se redujeron considerablemente en ratones tratados de acuerdo con el protocolo propuesto en este estudio y que utiliza líquido amniótico (LA) con contenido intacto de AFP. Esta histopatologia minima se debe posiblemente a dismi. nución de la reacción inflamatoria aguda del mesangio y a un "bloqueo" de la localización de leucocitos circulantes (PMN y MN) en los glomérulos, características de la glomerulitis inducida de acuerdo a los procedimientos de McCluskey y Benacerraf ${ }^{12}$. Aún más, los gloménulos de los animales tratados por 4 semanas con LA/AFP, mostraron una histopatologia no descrita hasta ahora y que consistía en una atenuación de la estructura glomerular, disminución de la extensión del mesangio, đistensión capilar y adelgazamiento de la pared capilar. Una estimación semicuantitativa sugirió una disminución del número de células presentes en los glomérulos. Estos hallazgos se correlacionaron estrechamente con la disminución o ausencia de depósitos inmunofluorescentes y densos en el mesangio, cuya matriz aparecía reducida. Estos cambios, sin embargo, requerirán confirmación y prueba de su reproducibilidad en otras cepas y especies. Es 
posible que las supuestas propiedades inmunosupresoras atribuidas a la AFP contenida en el LA hayan modulado la respuesta inflamatoria o inmunológica; sin embargo, el sitio de acción, local o sistémico, aún no ha sido determinado 29 . $60 \cdot 61 \cdot 64$.

Los estudios morfométricos en los grupos etarios descritos, documentaron la progresión de la mesangiopatía esclerosante y están de acuerdo con la información publicada por otros en ésta y otras cepas u otras especies de roedores $4 \cdot 13-23$ - $2426 \cdot 28 \cdot 58$. La esclerosis mesangial, acompariada inicialmente de hiperplasia, constituyó una lesión bien definida, alcanzó su máximo entre 9 y 12 a 14 meses de edad con progresión lenta a partir de este punto. Hubo una buena correlación entre los depósitos de IgG-IgM del mesangio e hilio glomerulares con los depósitos electrónicos densos. Debido a que un número importante de ratones de edad avanzada tenian IC circulantes, o substancias que de acuerdo con los procedimientos utilizados para su detecciôn se comportaban como tales, es posible que estos participen en el desartollo y progresión de la hiperplasia-esclerosis.

Estos resultados sugieren que se indujo una glomerulitis aguda en ratones jóvenes (machos) de la cepa $\mathrm{C} 57 \mathrm{~L} / \mathrm{J}$ mediante la inyección de IC solubles preformados, preparados en exceso de antígeno. Además, que la depuración de IC por el SRE estaba comprometida (disminuída) significativamente por el pretratamiento de los animales con LA que contenía AFP. Tercero, que el uso de LA del cual la mayor parte de la AFP habia sido extraída, no modificó la depuración de los IC. Cuarto, que posiblemente este efecto se deba a inhibición (directa o indirecta) del SRE, que no se asocio, como se esperaria, con una abundancia de IC en los tejidos. Aparentementé, la persistencia de los IC "in situ" no fue seguida de mayor acumulación sino que de degradación significativamente menor, que permitió detectar un aumento de la radioactividad tardia (2 hrs). Finalmente, estos estudios sugieren que el LA que contiene AFP protegió al riñón del efecto pro-inflamatorio de los IC; el mecanismo de esta acción, que probablemente resida en la AFP, no está aclarada.

\section{REFERENCLAS}

1. Cochrane, C.G.; Koffler, D.: Immune complex disease in experimental animals and man. Adv, Immunol. 16: 185, 1973.

2. Germuth, F.C.; Rodrigtez, E.: Immunopathology of the Renal Glomerulus, Little Brown and Company, Boston, 1973.

3. Glassock, R.J.; Bennetr, C.M.: The glomerulopathies. IN: The Kidney, Brenner B.M., Rector F.C.,
Jr., editors, p. 941, W.B. Saunders Co., Philadelphia, 1976.

4. Markham, R.V., Jr:; Sutheriand, J.C.; Mardiney, M.R.: The ubiquitous occurrence of immune complex localization in the renal glomeruli of normal mice. Lab. Invest. 29: 111, 1973.

5. Nyedegger, U.E.: Biologial properties and detection' of immune complexes in animal and human pathology. Rev. Physiol. Biochem. Phamac. 185: 63, 1979.

6. Stensgaard, J., Johansen, A.S.: Biochemical aspects of immune complex fomation and immune complex disease. Allergy 35: 457, 1980 .

7. Urizar, R.E.; Giboa, N.; Largent, J.A.: Immunologic mechanisms of childhood renal diseases. Paediatrician 10: 254, 1981 .

8. Couser, W.G.; Salant, D.J.: In siru immune complex fomation and glomerular injury. Kidney lnt. 17: 1, 1980 .

9. Fleuren, G.; Grond, J., Hoedemaeker, P.J.: In situ formation of subepithelial glomerular immune complexes in passive serum sickness. Kidney Int. 17: 631,1980 .

10. Gallo, G.; Cauin-Glasser, T.; Lamm, M.E.: Charge of circulating immune complexes as a factor in glomerular basement membrane localization in mice. J. Clin. Invest. 67: 1305, 1981.

11. Theophilopoulos, A., Dixon, F.J.: Immune complexes in human disease. Am. J. Pathol. 100: $\$ 29,1980$.

12. McCluskey, R.J.; Benacerraf, B.: Localization of colloidal substances in vascular endothelium. A mechanism of tissue damage. Il. Experimental serum sickness with acute glomerulonephritis induced passively in mice by antigen-antibody complexes in antigen excess. Am. J. Pathol. 35: $275,1959$.

13. Couser, W.G.; Stillmant, M.D.* Mesangial lesions and focal glomerular sclerosis in the aging raf. Lab. Invest. $33: 491,1975$.

14. Mannit, $M$. : Physiochemical and functional relationships of immune complexes, J. Invest. Dermatol. 74: 333, 1980.

15. Barcelli, V.; Rademacher, R.; Ooi, Y.M,; Doi, B.S.: Modification of glomerular immune complex deposition in mice by activation of the reticuloendothelial system. J. Clin. Invest. 67: 20, 1981.

16. Benacerraf, B.; Sebastyrn, M.; Cooper, N.S.: The clearance of antibody complexes from the blood by the reticuloendothelial system. J. Immunol. 82: $131,1959$.

17. Finblood, D.S.; Abeles, D.; Rifai, A., Plotz, P.H.: The specificity of uptake of model immune complexes and other protein aggregates by the murine reticuloendothelial system. J. Immunol 125:1060, 1980.

18. Finbloom, D.S. Lorz, P.H.: Studies of reticuloendothelial function in the mouse, with model immune complexes. L. Serum clearance. J. Immunol. 123: 1594, 1979 :

19. Michael, A.F,; Keane, W.F.; Raij, L.; Mauer, S.M.: The glomerular mesangium. Kidney Int. 17: 141, 1980 .

20. Raij, L., Sibley, R.K., Keane, W.: Mononuclear phagocytic system stimulation. Protective role for glomerular immune complex deposition. J. Lab. Clin. Med. 98: 55B, 1981.

21. Bolton W.K., Benton F.R., Maclay J.S., Sturgill B.S.: Spontaneous glomerular sclefosis in aging Sprague-Dawley rats. I. Lesions associated with 
mesangial IgG deposits. Am. J. Pathol. 85: 277 , 1976.

22. Boiton W.X., Stugill B.C.: Spontaneous glomerular sclerosis in aging Sprague-Dawley rats. II. Ultrastructural studies. Am. J. Pathol. 98: 339, 1980.

23. Elemg J.D., Arends A.: Focal and segmental glomerular hyalinosis and sclerosis in the rat. Lab. Invest. 33: 554,1975 .

24. Gutmon P.H., Wepper K.D., Fudenberg H.H.: On the presence of $\operatorname{lgG}$ and $\mathrm{Blc}$ globulins in renal glomeruli in aging and neonatally irradiated mice. Vox Sang 12: 329, 1967.

25. Kreisberg J.L., Kamovsky M.J.: Focal glomerular sclerosis in the fawn hooded rat. An. I. Patol. 92: 637,1978

26. Linder E., Pasternack A., Edgington T.S.: Pathology and immunology of age-associated disease of mice and evidence for an autologous inmune complex pathogenesis of the associated renal disease. Clin. Immunol. Immunopathol. 1: 104, 1972.

27. Peter C.P.: Possible immune origin of age-related pathological changes in long-lived mice. J. Gerentol. $28: 265,1973$

28. Porter D.D., Porter H.G, Cos N.A.: Immune complex glomerulonephritis in one year old $C 57 \mathrm{~L} / \mathrm{J}$ mice induced by endogenous murine leukenia virus. J. Immunol. 111 : 1626, 1973.

29. Tomasi T.B., Jr., Dotweyler R.J., Murguita R.A., Keller R.H.: Immunosuppression by alphafetoprotein. Trans. Assoc. Am. Physicians 88: 293, 1975 .

30. Himmelhoch S.R.: Chromatography of proteins on ion-exchange adsorbents. Meth. Enzymol. 22: 273, 1971 .

31. Peterson E.A., Sober H.A.: Chromatography of proteins. [. Cellulose ion-exchange adsorbent. J. Am. Chem. Soc. 78: 751, 1956.

32. Hubband A.L., Cohn Z.A.: The enzymatic jodination of the red cell membrane. J. Cell Biol. 55:390, 1972.

33. Marchalonis J.J.: An enzymatic method for the trace iodination of immunoglobulins and other protejns. Biochem J. 113: 299, 1969.

34. Mizejewsky G.J., Plummber J.M., Blanchette K.A., Vonnegut $M$. ., Jacobsen H.I. Alphafetoprotein: Immunoreactivity of the major estrogen-binding component in mouse amniotic flujd. Immunol. 36: $685,1979$.

35. Ouchterlony 0 .: Handbook of Immunodiffusion and Immunoelectrophoresis (1st edition), Ann Arbor Science, Ann Arbor, M.I., pp. 21-31, 1968.

36. Lowry O.H., Rosebrough N.F., Farr A.L., Randell $R . J .:$ Protein measurement with the folin phenol rcagent. J. Biol. Chem. 193:265, 1951.

37. Mizejewsky G.J., Allen R.P.: Immunotherapeutic suppression in transplantable solid tumors. Nature (London) 250: 50, 1974.

38. Mizejewsky G.J., Young R., Allen R.P.: Alphafetoprotein: Effect of heterologous antiserum on hepatoma cells in vitro. J. Natl. Cancer Inst. 54 : $1361,1975$.

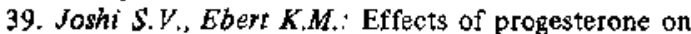
labelling of soluble proteins and glycoproteins in rabbit endometrium. Fertil Steril 27: 730, 1976

40. Mizejewsky G.J., Vonnegut M., Simon R.: Estradiol affinity chromatography. Application to purifica. tion of murine alphafetoptotein. J. Chromatogra. phy 202: 113, 1980

41. Creighton W.D., Lambent P.H., Miescher P.A.: Detection of antibodies and soluble antigen-antj. body complexes by precipitation with polyethylene glycol. J. Immunol. 111: 1219, 1973.

42. Myllyala G.: Aggregation of human blood platelets by immune complexes in the sedimentation pattern test. Scand. J. Hematol. (Suppl) 19: 1-56, 1973.

43. Urizar R.E., Sherer G., Tartaglai A., et al.: Disseminated intravascular coagulation induced by liquoid in the rat. I. Correlation of hematologic and complement abnormalities with renal lesions studied by light fluorescence and electron microscopy. Lab. Invest. 32: 270, 1975

44. Urizar R.E., Tinglof B.D., Smith F.G., et ol.: Persistent asymptomatic proteinuria in children. Functional and ultrastructural evaluation with special reference to glomerular basement membrane thickness. Am. J. Clin. Pathol. 62: 461, 1974.

45. Toylor R.N., Juong A.Y., Fulford K.M.: Quality control for immunologie tests. USDHHS publication (CDC) 79: 8379, 1979 .

46. Fleiss $J$.: Statistical methods for rates and proportions. J. Wiley and Sons, New York, 1973.

47. Ford P.M.: Glomerular localization of aggregated protein in mice: Effect of strain differences and relationship to systemic macrophage function. Br. J. Exp. Pathol. 56: 307, 1975.

48. Ford P.M.: Passive serum sickness in the mouse: Effect of interstrain differences on glomerular deposition of immune complexes. Br. J. Exp. Pathol. 56: 199, 1975.

49. Hoffsten P.E., Swerdlin A., Bartell M., Hill C.L., Venverloh J., Broth arson K., Klaht S.; Reticuloendothelial and mesangial function in murine immune complex glomerulonephritis. Kidney Int. 15: 144, 1979.

50. Haakenstad A.O., Mannik $N$.: Saturation of the reticuloendothelial system with soluble immune complexes. J. Immunol. 112; 139, 1974.

51. Haakensfad A.O., Case J.B., Mannik M.: Effect of cortisone on the disappearance kinetics and tissue localization of soluble immune complexes. J. Immunol. 114: 1153, 1975.

52. Shvil Y., Michael A.F., Mater S.M.: Uptake of ggregated immunoglobulin by the mouse kidney. I. Effect of endotoxin. Br. J. Exp. Pathol. 61: 22, 1980 .

53. Shvil Y., Mauer S.M. Michael A.F.: Uptake of aggregated immunoglobulin by the mouse kidney. II. Effect of hydrocrotisone. Br. J. Exp. Pathol. 6l : 30,1980 .

54. Elems J.D., Hoyer J.R., Vemier R.L.: The glomerular mesangium: Uptake and transport of intravenously injected colloidal carbon in rats. Kidney Int. 9: 396, 1976

55. Lee S., Vernier R.L.: Immunoelectron microscopy of the glomerular mesangial uptake and transport of aggregated human albumin in the mouse. Lab. Invest. 42: 44, 1980.

56. Mauer S.M., Fish A.J., Blou E.D., Michne! A.F.: The glomerular mesangium. l. Kinetic studies of macro. molecular uptake in nomal and neoplastic rats. $J$. Clin. Invest. 51: 1092, 1972

57. Striker G., Mannik M., Tuny M.Y.: Role of marrow derived monocytes and mesangial cells in removal of immune complexes from renal glomeruli. J. Exp. Med, 149: 127, 1979.

58. Okumura $K$, Kondo $Y$, , Tada $T$.: Studies on passive serum sickness. I. The glomerular fine structure of serum sickness nephritis induced by preformed antigen-antibody complexes in the mouse. Lab. Invest. 24: 383, 1971 . 
59. Murgita R.A., Tomasi T.R., Jr.: Suppression of the immune response by alphafetoprotein. I. Effect of mouse alphafetoprotein in the primary and secondary antibody response. J. Exp. Med. 141: 269, 1975.

60. Murgito R.A., Tomasi T.B., Jr.: Suppression of the immune response by alphafetoprotein. Il. The effect of mouse alphafetoproteiz on mixed lymphocyte reactivity and mitogen induced lymphocyte transformation. J. Exp. Med. 1412: 440, 1975.

61. Murgito R.A., Goidl E.A., Kontiainen S., Beverley P.C.L. Wigzell H.: Alphafetoprotein induces suppressor $\mathrm{T}$-cells in vitro. Nature 267: 257-259, 1977.

62. Murgita R.A., Anaersson L.C., Sherman' M.S.,
Bennich $H$., Wigzell $H$ :- Effects of human atphafetopzotein on human $B$ and $T$ lymphocyte proliferation in vitro. Clin. Exp. Immunal. 33: 347, 1978.

63. Tomast T.B., J.: Structure and function of alphafetoprotein. Ann. Rev. Med. 2B: 453, 1977.

64. Tomasi T.B., Jr.: Suppressive factors in amniotic fluid and newbom serum: Is alphafetoprotein involved? Cell. Immunol. 37: 459, 1978.

65. Yazova A., Suslov A.P., Brondz B.D.. Temination of natural tolerance to alpha-fetoprotein in rats: Study of cell-mediated immunity in the macrophage migration inhibition test. (T-B cell separation/peritoneal exudate cells.) Folis Bilogic (Praha) 24: 81, 1978. 